\title{
A COMPARATIVE STUDY OF THE MOST WIDELY HARVESTING SYSTEMS FOR WHEAT CROP IN EGYPT
}

\section{Mohamed Sayed Omran*}

\begin{abstract}
\end{abstract}
Field experiments were carried out at the experimental field of Rice Mechanization Center, Meet El-Deeba, Kafer El-Sheikh Governorate during harvesting season of 2007.Five of the most widely wheat harvesting systems were studied on the widely common wheat cultivar (Sakha93): multi-purpose combine harvester, through in combine harvester, hold in combine harvester, Reaper + thresher and double knives mounted mower + thresher to recommend the appropriate system for harvesting wheat crop. The systems were evaluated according to the technical parameters: actual performance rate, field efficiency; cutting efficiency, cleaning efficiency; percentage of the total losses and consumed energy, according to (RNAM 1995) and according to the financial criteria. Overall cost criterion was used to evaluate the tested wheat harvesting systems.

The results showed that the combine harvester realized the highest actual performance rate $(3.06 \mathrm{fed} . \mathrm{h})$ at all the tested forward and threshing speeds compared with the other studied systems. The highest field efficiency (94.3\%) was obtained from the $4^{\text {th }}$ system and the $5^{\text {th }}$ system. The highest cutting efficiency (94.3\%) was conducted by hold in combine harvester. The highest cleaning efficiency $(98.7 \%)$ resulted from $4^{\text {th }}$ and $5^{\text {th }}$ systems at threshing drum speed $35.34 \mathrm{~m} / \mathrm{s}$. The lowest percentage of total grain losses averaged $(2.27 \%)$ resulted from the $3^{\text {ed }}$ system. The lowest consumed energy per fed and per ton was achieved by multipurpose combine harvester. The lowest significant total cost $(85.72 \mathrm{LE} / \mathrm{h})$ resulted from $4^{\text {th }}$ system.

The overall cost criterion (LE/fed.) was observed from harvesting systems; it includes actual performance rates, consumed energy, cost of total losses grain, and total costs. The lowest value is considered the most appropriate from operation of multi-purpose combine harvester.

* Assist . Prof., Ag. Eng. Dept. , Fac . of Ag., Cairo University. 


\section{INTRODUCTION}

$\mathrm{W}$ heat is one of the most important grain crops in Egypt, it occupies about 2.985 million feddans with a national average of 2.71 ton/fed.( G.M.A.S 2006) .As known, cereal crops are too sensitive to harvesting operation due to the high percentages of grain losses affecting the total yield, there fore much care has to be taken during harvesting operation to minimize production losses and hence costs.

Ghonimy and Rostom (2002) developed an overall criterion for evaluating four types of head feeding combines (YANMAR CA-32, YANMAR CA-385EG, KUBOTA R1-40 and KUBOTA PRO-48). It depends upon the relative weight for each technical and economical evaluation criterion. This overall criterion is suitable for mechanization services suppliers by using (operating hour costs) as economical evaluation parameter and suitable for the farmers (mechanization services users) by using (renting costs) as economical evaluation parameter. The combines arrangement according to the final overall criterion for the farmers (mechanization services users) were PRO-48, CA-385EG, R1-40, and CA-32. El-Sahrigi and Khan (1990) reported that reapers have been used for harvesting wheat and rice, however, the output of the reaper was low and considerable labor was still required for collection, threshing, and bagging seeds. Mohamoud et al. (2007) developed the feeding device of the Turkish thresher to be suitable for threshing wheat crop with high efficiency. The obtained data showed that the developed device decreased un-threshed grains by $26.99 \%$, damaged grains by $40.37 \%$ total grain losses by $38.85 \%$ and threshing cost by $14.27 \%$. In addition threshing efficiency, cleaning efficiency and energy requirement were increased by $0.62 \%, 3.00 \%$ and $3.49 \%$ respectively, at feeding rate of $1.1 \mathrm{ton} / \mathrm{h}$, drum speed of $27 \mathrm{~m} / \mathrm{s}$ and grain moisture content of $19 \%$.

Arnaout et al. (1998) investigated some different mechanizing systems for harvesting wheat and rice crops under Egyptian conditions. They found that in wheat crop:

- The minimum grain losses $(1.66 \%)$ resulted from wheat combine. 
- The highest efficiency (78.04\%) was obtained under self-propelled mower.

- The highest field capacity $(1.64 \mathrm{fed} / \mathrm{h})$ was remarked under reaper.

- The minimum energy (25.38 kWh/fed) was consumed under selfpropelled mower + threshing by threshing machine.

- The minimum total costs (173.03 L.E/fed) was obtained under combines.

Kamel (1999) used two different types of Yanmar combines (CA- 385 and CA-760) to harvest three rice varieties (Giza 178, Sakha 101, and Sakha 102). He stated that all kinds of losses for the two combines increased with the increase of harvesting speed and cutting height for the three selected rice varieties. He added that the lowest value of total losses obtained at harvesting forward speed $0.3 \mathrm{~m} / \mathrm{s}(1.08 \mathrm{~km} / \mathrm{s})$ with cutting height $7 \mathrm{~cm}$. He also mentioned that the highest value of total losses for both two types of combines did not exceed $5.8 \%$ compared with $25 \%$ when utilizing traditional harvesting system. Afify et al. (2000) mentioned that four harvesting systems (manual + thresher, tractormounted mower + thresher, Deutz fahr combine, and Yanmar combine) with three planting methods (manual transplanting, drilling, and mechanical transplanting) were tested to select the proper system of rice harvesting which suits the planting method. They found that the least costs of planting, harvesting, and percentage of losses were 104.21 LE/fed., $84 \mathrm{LE} /$ fed. and $2.18 \%$ respectively were obtained by using of drilling system and harvesting by Deutz Fahr combine. El-wady et al. (2000) evaluated the performance of Barmel rice-thresher. They concluded that the best performance of Barmeel rice thresher was at 550 rpm $(29.64 \mathrm{~m} / \mathrm{s})$ which gave minimum criterion cost, acceptable cleaning efficiency of $94.61 \%$ and threshing capacity of $2.68 \mathrm{ton} / \mathrm{h}$. El-Nakib et al. (2003) used Kubota combine as a mechanical harvester of rice crop (Sakha 102). They found that header, threshing, separating and shoe losses increased with the increase of the forward speed and the decrease of grain moisture content. The optimum operating parameters for harvesting rice crop were combine forward speed of $4.5 \mathrm{~km} / \mathrm{h}$ and grain moisture content of $16.5 \%$. El-Khateeb (2005) tested multi-purpose combine harvester (Yanmar model CA-760) for harvest rice crop, and 
found that the maximum value of actual field capacity was $2.90 \mathrm{fed} / \mathrm{h}$ at forward speed of $3.0 \mathrm{~km} / \mathrm{h}$ and grain moisture content of $18 \%$. Also, he found that the highest value of fuel consumption rate was $7.20 \mathrm{~L} / \mathrm{fed}$ at forward speed of $1.5 \mathrm{~km} / \mathrm{h}$ and grain moisture content of $25 \%$. He recommended that grain moisture content of $22.0 \%$, forward speed of $1.5 \mathrm{~km} / \mathrm{h}$, cylinder speed of $24.0 \mathrm{~m} / \mathrm{s}$ were the optimum operating conditions for mechanical harvesting rice crop. Also, using combine harvester was the most efficient and economic system (89.70 L.E/fed) compared to manual harvesting and gathering followed by threshing and winnowing (181.60 L.E/fed). El-Sharabasy (2007) mentioned that using both full and partial mechanization system for harvesting and threshing rice crop at the higher forward speeds and lower grain moisture contents, recorded minimum consumed energy and cost requirements. Also using partial or full mechanization for harvesting rice crop saved time, effort, and total cost requirements and also cleared the rice crop from the field as fast as possible than traditional manual system.

Selection of the optimum system to harvest wheat crop and improving of the combine performance during harvesting are very important to minimize both grain losses and operating costs. Therefore, the present study aimed to compare between the most widely wheat harvesting systems to recommend the appropriate system to harvest wheat crop based on some technical and financial parameters.

\section{MATERIAL AND METHODS}

This study was carried out during 2007, and was conducted in the experimental field of Rice Mechanization Center, Meet El-Deeba, Kafer El-Sheikh Governorate. Using the most widely systems to harvest wheat crop (Sakha 93). This site was chosen since Kafer El-Sheikh Governorate is considered one of the four highest governorates in Egypt in terms of wheat grain cultivated area (216871 fed.) and average productivity 2.781 ton/fed. (G.M.A.S 2006)

\section{The components of the wheat harvesting systems are:}

1- Multi-purpose combine harvester (Class dominator 68S )

2- Through in combine harvester (Yanmar CA-760)

3- Hold in combine harvester (Yanmar CA-3850)

4- Reaper AR 120 + Thrasher(Mabrouk) 
5- Double knife mounted mower (Posates ) + Thresher(Mabrouk)

Table (1) shows the technical specifications of the components of the wheat harvesting systems

\section{Treatments:}

\section{1- System types:}

Five types of harvesting systems which were mentioned above were studied.

\section{2- Forward speed:}

Three tested forward speeds for $1^{\text {st }}$ system ( $2.3,3.2$ and $4.5 \mathrm{~km} / \mathrm{h}$ ), two tested forward speeds for $2^{\text {ed }}$ and $3^{\text {ed }}$ systems $(1.8$ and $3.2 \mathrm{~km} / \mathrm{h}$ ), two tested forward speeds for $5^{\text {th }}$ system $(2.1$ and $3.2 \mathrm{~km} / \mathrm{h})$ and one tested forward speeds for $4^{\text {th }}$ system $(1.8 \mathrm{~km} / \mathrm{h})$.

\section{3- Threshing cylinder speed:}

Two threshing cylinder speeds for every forward speed were used.

The treatment symbols are showen in table (2).

\section{Measurements:}

The combines were operated at the similar optimum condition. Class combine was operated with three levels of forward speeds, while Yanmar combines were operated with two levels of forward speeds as they have only two forward gear synchronizations for harvesting and high gear synchronizations speed for road operation. For each of the tested forward speeds, the most common two threshing cylinder speeds were examined. The threshers were operated with the experience recommended three levels of thresher cylinder speeds with the recommended feeding rate $0.35 \mathrm{~kg} / \mathrm{s}$, by dividing the plants into $7 \mathrm{~kg}$ bunches and feeding it to the thresher every 20 seconds.

Actual performance rate, field efficiency, threshing efficiency; cutting efficiency, percentage of damaged grains, percentage of lost grains, cleaning efficiency, specific consumed energy and total costs (according to $R N A M, 1995$ ) were used to evaluate the tested systems.

\section{1- Actual performance rate (Pr)}

$$
\mathrm{P}_{\mathrm{r}}=\mathrm{H}_{\mathrm{a}} / \mathrm{T}_{\mathrm{c}}
$$

Where:

$\mathrm{H}_{\mathrm{a}}=$ total harvested area, fed.;

$\mathrm{T}_{\mathrm{c}}=$ total consumed time, $\mathrm{h}$. 
The total consumed time for $4^{\text {th }}$ and $5^{\text {th }}$ systems was related to the time consumed for threshing operation. Since threshing operation per feddan required longer time than that required for harvesting.

\section{2- Field efficiency $\left(\boldsymbol{\eta}_{\mathbf{t}}\right)$}

The field efficiency was calculated from the following formula

$$
\eta_{\mathrm{t}}=\left\{\left(\mathrm{T}_{\mathrm{h}}-\mathrm{T}_{\mathrm{u}}\right) / \mathrm{T}_{\mathrm{h}}\right\}^{*} 100
$$

Where:

$\mathrm{T}_{\mathrm{h}}=$ total time for harvesting and threshing processes per fed., $\mathrm{h}$;

$\mathrm{T}_{\mathrm{u}}=$ total un-productive time during harvesting and threshing process per fed., h.

\section{3- Cutting efficiency $\left(\eta_{c}\right)$}

An average length of 100 plants from different 10 locations in the field during and after harvesting were measured to calculate cutting efficiency.

$$
\eta_{\mathrm{c}}=\left(\mathrm{h}_{\mathrm{h}} / \mathrm{h}_{\mathrm{t}}\right) * 100
$$

Where:

$\mathrm{h}_{\mathrm{h}}=$ average height of plant after cutting, $\mathrm{cm}$;

$\mathrm{h}_{\mathrm{t}}=$ average height of plant before cutting, $\mathrm{cm}$.

\section{4- Cleaning efficiency $\left(\boldsymbol{\eta}_{\mathrm{cl}}\right)$}

$$
\eta_{\mathrm{c}}=(\mathrm{Wg} / \mathrm{Mo})^{*} 100
$$

Where:

$\mathrm{Wg}=$ weight of whole output grain per unit area (threshed, un-threshed and damaged grain), ton/fed

Mo = weight of all output materials per unit area, ton/fed.

\section{5 - Percentage of damaged $\left(P_{d}\right)$ un-threshed grains ( $\left.U_{t h}\right)$ :}

Visual investigation and manual separation of 10 samples each of 100 grams were used to calculate percentage of damaged and un-threshed grains.

$$
\mathbf{P}_{\mathbf{d}}=(\mathrm{D} / \mathrm{A}) * 100
$$

Where:

$\mathrm{D}=$ average of damaged grains weight of 10 samples, $\mathrm{g}$.

$\mathrm{A}=$ sample weight $=100 \mathrm{~g}$.

$\mathbf{U}_{\mathbf{t h}}=(\mathrm{E} / \mathrm{A}) * 100$

$\mathrm{E}=$ average of un-threshed grains weight of 10 samples, $\mathrm{g}$. 


\section{6- Percentage of grain losses $\left(\mathbf{P}_{\mathrm{gl}}\right)$}

Grain yield was estimated by manual harvesting 5 plots each of $(1 \times 1 \mathrm{~m})$ with high care from random locations. The harvested plants were manually threshed; the threshed grains were weighed for each sample.

The Percentage of grain losses was calculated from equation (7):

Where:

$$
\mathrm{Pgl}=\{(\mathrm{Gy}-\mathrm{Wg}) \div \mathrm{Gy}\} * 100
$$

Gy = weight grain yield, ton/fed.

\section{7-Percentage of total grain losses (Tgl):}

$\mathrm{Tgl}=\mathrm{P}_{\mathrm{d}}+\mathrm{U}_{\mathrm{th}}+\mathrm{Pgl}$

\section{8- Specific consumed energy (Se )}

$\mathrm{Se}=3.163 \mathrm{Fc} / \mathrm{Ap}, \mathrm{kW} . \mathrm{h} /$ ton

Where:

$3.163 \mathrm{Fc}=$ the required power, $\mathrm{kW}(3.163=$ conversion constant, according to Omran, 1989).

$\mathrm{Fc}=$ fuel consumption, $\mathrm{L} / \mathrm{h}$.

Ap $=$ Actual system productivity $=\mathrm{Wg} * \mathrm{P}_{\mathrm{r}}$, ton $/ \mathrm{h}$

\section{9- Total Cost (Tc)}

The total costs (Tc) were estimated using equation of Oida 1997 according to 2008 price levels.

$$
T C=\frac{\left\{\left[\frac{P-s}{Y}+\left[\frac{P+S}{2} \cdot \frac{i}{100}+(0.02 P)\right]+\left[\left(\frac{P . r}{Y}\right)+(f P . F c . n)+(\text { Oc.C.n })+(N . L . n)\right]\right]\right\}}{n} \ldots . . .(3)
$$

where:

Tc $=$ Total cost, LE/h;

$P=$ Purchase price, LE;

$=370000$ LE for Class ;250000 LE Yanmar CA-760 ; 160000 LE Yanmar CA-385 ; 29000 LE Reaper AR 120 ; 15500 LE Thrasher AS75, 12300 LE Thrasher Mabrouk, 7500 LE Posates mower and 54000 for tractor.

$S$ = Salvage value, LE;

$=0.68(0.920)^{Y * P}$ for tractor and self prepared machine

$=0.65(0.885)^{Y * P}$ for machine

$i=$ Interest rate, $\% ;=9 \%$

$r=$ Coefficient of repair and maintenance $=0.80$, decimal; 


$$
\begin{aligned}
Y & =\text { Anticipated length of time owned, Year; } \\
& =5 \text { years for the machine, and } 10 \text { years for the tractor. } \\
f p & =\text { Fuel price, LE/lit; } \\
& =1.1 \mathrm{LE} / \text { lit for Diesel fuel, } 1.75 \mathrm{LE} / \text { lit for Gasoline fuel } \\
F c & =\text { Fuel consumption, lit/h; } \\
O c & =\text { Oil consumption }=0.00059 * P t+0.02169, \mathrm{Lit} / \mathrm{h} ; \\
P t & =\text { Engine power }, \mathrm{HP} \\
C & =\text { Oil price, LE/lit; } \\
& =15 \mathrm{LE} / \mathrm{lit} ; \\
N & =\text { Number of labors } \\
L & =\text { Labor wage, } \mathrm{LE} / \mathrm{h} ;
\end{aligned}
$$

The common labour wages of Kafer El-Sheikh Governorate were: $10 \mathrm{LE} / \mathrm{h}$ for combine operator,7 LE/h for tractor operator, $5 \mathrm{LE} / \mathrm{h}$ for technical labor, $4 \mathrm{LE} / \mathrm{h}$ for labor

-The $1^{\text {st }}, 2^{\text {nd }}$ and $3^{\text {rd }}$ systems require combine operator and two labors for collecting grains and handling grain sacs.

-The $4^{\text {th }}$ system requires one technical labor for operating reaper and 5 labors for collecting harvested plants, threshing and handling.

-The $5^{\text {th }}$ system requires one tractor operator, one technical labor and 7 labor (2 labors for collecting harvested plants and 5 labors for threshing harvested plants and grain handling.

$n$ = Yearly working hours, $\mathrm{h} /$ year.

$=500$ for the machine and 1000 for tractor.

\section{0 - Overall cost criterion:}

In order to select an overall criterion for evaluating the tested systems of wheat harvesting, the cost of total grain losses (LE/fed.) was determined and added to the total costs of the system (LE/fed.). The price of one ton wheat grain was equivalent to $2600 \mathrm{LE}$. (2008 prices). The total cost (LE/fed.) includes performance rate and field efficiency while the energy consumed expressed as consumed fuel in the operating cost.

11- Statistical analysis was carried out for the obtained data using. MSTAT program to compare between the performances of the studied harvesting systems using randomize complete block design (LSD of $\boldsymbol{P}$ 0.05) with three factors (harvesting systems, forward speeds and threshing cylinder speeds). 
Table (1) Summarized of the technical specifications of the harvesting systems components

\begin{tabular}{|c|c|c|c|c|c|c|c|}
\hline Item & $\begin{array}{c}\text { Class } \\
68 S\end{array}$ & $\begin{array}{l}\text { Yanmar } \\
\text { CA-760 }\end{array}$ & $\begin{array}{l}\text { Yanmar } \\
\text { CA-385 }\end{array}$ & $\begin{array}{l}\text { Walking } \\
\text { Reaper } \\
\text { AR 120 }\end{array}$ & $\begin{array}{c}\text { Tractor } \\
\text { Naser } 65\end{array}$ & $\begin{array}{l}\text { Thresher } \\
\text { (Mabrouk) }\end{array}$ & $\begin{array}{l}\text { Mower } \\
\text { Pozates }\end{array}$ \\
\hline $\begin{array}{l}\text { Overall dimensions: } \\
\text { Length, } \mathrm{cm} \\
\text { Width, } \mathrm{cm} \\
\text { Height, } \mathrm{cm}\end{array}$ & $\begin{array}{l}960 \\
590 \\
354\end{array}$ & $\begin{array}{l}560 \\
243 \\
265\end{array}$ & $\begin{array}{l}406 \\
190 \\
216\end{array}$ & $\begin{array}{l}130 \\
110 \\
120\end{array}$ & $\begin{array}{l}345 \\
190 \\
240\end{array}$ & $\begin{array}{l}415 \\
127 \\
198\end{array}$ & $\begin{array}{c}110 \\
230 \\
80\end{array}$ \\
\hline $\begin{array}{l}\text { Cutting device: } \\
\text { Cutting width, } \mathrm{cm} \\
\text { Cutting adjusted }\end{array}$ & $\begin{array}{c}420 \\
\text { Hydraulically }\end{array}$ & $\begin{array}{c}206 \\
\text { Hydraulically }\end{array}$ & $\begin{array}{c}140 \\
\text { Hydraulically }\end{array}$ & $\begin{array}{c}100 \\
\text { Manually }\end{array}$ & 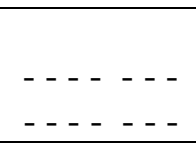 & $\begin{array}{l}-- \\
-\end{array}-$ & $\begin{array}{c}120 \\
\text { Hydraulically }\end{array}$ \\
\hline $\begin{array}{l}\text { Threshing unit type } \\
\text { Threshing drum: } \\
\begin{array}{l}\text { Diameter, cm } \\
\text { Length, } \quad \mathrm{cm}\end{array}\end{array}$ & $\begin{array}{l}\text { Straw walker, } \\
\text { shaking, } \\
\text { sieves \& fans } \\
\\
45 \\
106\end{array}$ & $\begin{array}{c}\text { Screw rotor } \\
\\
\\
65 \\
217\end{array}$ & $\begin{array}{c}\text { Shaking } \\
\text { sieves \& fans } \\
\\
42 \\
71\end{array}$ & 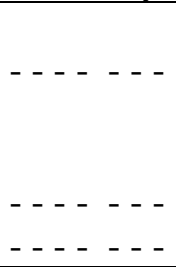 & 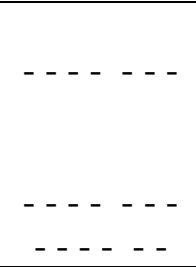 & $\begin{array}{c}\text { Beater type with } \\
\text { bolted flat } \\
\text { fingers } \\
75 \\
118\end{array}$ & $\begin{array}{c}----- \\
\\
----- \\
-----\end{array}$ \\
\hline $\begin{array}{l}\text { Engine: } \\
\text { Power/motor speed, } \\
\text { HP / rpm } \\
\text { No. of cylinders } \\
\text { Used fuel }\end{array}$ & $\begin{array}{c}105 / 2500 \\
4 \\
\text { Diesel }\end{array}$ & $\begin{array}{c}58 / 2300 \\
4 \\
\text { Diesel }\end{array}$ & $\begin{array}{c}38 / 2000 \\
3 \\
\text { Diesel }\end{array}$ & $\begin{array}{c}5 / 550 \\
1 \\
\text { Gasoline }\end{array}$ & $\begin{array}{c}62 / 2300 \\
4 \\
\text { Diesel }\end{array}$ & 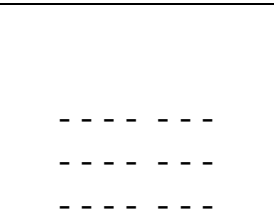 & 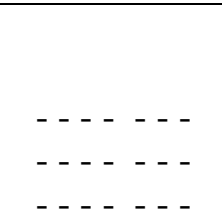 \\
\hline Traction system & $\begin{array}{l}\text { Rubber } \\
\text { crawler }\end{array}$ & $\begin{array}{l}\text { Rubber } \\
\text { crawler }\end{array}$ & $\begin{array}{l}\text { Rubber } \\
\text { crawler }\end{array}$ & $\begin{array}{c}2 \text { Rubber } \\
\text { wheals }\end{array}$ & $\begin{array}{c}2 \times 4 \text { Rubber } \\
\text { wheals }\end{array}$ & Rubber wheal & - - - - - - \\
\hline
\end{tabular}

Table ( 2 ): Treatments symbols of the used harvesting systems 


\begin{tabular}{|c|c|c|c|c|c|c|c|c|c|c|c|c|c|c|c|c|c|c|c|c|c|c|c|c|c|c|}
\hline No & System & $\begin{array}{c}\text { Forward } \\
\text { Speeds, } \\
\text { km/ h }\end{array}$ & $\begin{array}{c}\text { threshing } \\
\text { Speeds, } \\
\text { m /s }\end{array}$ & $\mathbf{A}$ & B & C & D & $\mathbf{E}$ & $\mathbf{F}$ & $\mathbf{G}$ & $\mathbf{H}$ & I & $\mathbf{J}$ & $\mathbf{K}$ & $\mathbf{L}$ & $\mathbf{M}$ & $\mathbf{N}$ & $\mathbf{O}$ & $\mathbf{P}$ & $\mathbf{Q}$ & $\mathbf{R}$ & $\mathbf{S}$ & $\mathbf{T}$ & $\mathbf{U}$ & $\mathbf{W}$ & $\mathbf{Y}$ \\
\hline \multirow{6}{*}{1} & \multirow{6}{*}{$\begin{array}{l}\text { Class } \\
68 \mathrm{~S}\end{array}$} & \multirow{2}{*}{2.3} & 28.90 & $*$ & & & & & & & & & & & & & & & & & & & & & & \\
\hline & & & 32.04 & & $*$ & & & & & & & & & & & & & & & & & & & & & \\
\hline & & \multirow{2}{*}{3.2} & 28.90 & & & $*$ & & & & & & & & & & & & & & & & & & & & \\
\hline & & & 32.04 & & & & $*$ & & & & & & & & & & & & & & & & & & & \\
\hline & & \multirow{2}{*}{4.5} & 28.90 & & & & & $*$ & & & & & & & & & & & & & & & & & & \\
\hline & & & 32.04 & & & & & & $*$ & & & & & & & & & & & & & & & & & \\
\hline \multirow{4}{*}{2} & \multirow{4}{*}{$\begin{array}{c}\text { Yanmar } \\
\text { C760 }\end{array}$} & \multirow{2}{*}{1.8} & 22.70 & & & & & & & $*$ & & & & & & & & & & & & & & & & \\
\hline & & & 32.10 & & & & & & & & $*$ & & & & & & & & & & & & & & & \\
\hline & & \multirow{2}{*}{3.2} & 22.70 & & & & & & & & & $*$ & & & & & & & & & & & & & & \\
\hline & & & 32.10 & & & & & & & & & & $*$ & & & & & & & & & & & & & \\
\hline \multirow{4}{*}{3} & \multirow{4}{*}{$\begin{array}{c}\text { Yanmar } \\
\text { CA385 }\end{array}$} & \multirow{2}{*}{1.8} & 22.70 & & & & & & & & & & & $*$ & & & & & & & & & & & & \\
\hline & & & 32.10 & & & & & & & & & & & & $*$ & & & & & & & & & & & \\
\hline & & \multirow{2}{*}{3.2} & 22.70 & & & & & & & & & & & & & $*$ & & & & & & & & & & \\
\hline & & & 32.10 & & & & & & & & & & & & & & $*$ & & & & & & & & & \\
\hline \multirow{3}{*}{4} & \multirow{3}{*}{$\begin{array}{c}\text { Reaper } \\
\text { AR 120 + } \\
\text { Thresher } \\
\text { Mabrouk }\end{array}$} & \multirow{3}{*}{1.8} & 27.48 & & & & & & & & & & & & & & & $*$ & & & & & & & & \\
\hline & & & 31.41 & & & & & & & & & & & & & & & & $*$ & & & & & & & \\
\hline & & & 35.34 & & & & & & & & & & & & & & & & & $*$ & & & & & & \\
\hline \multirow{6}{*}{5} & \multirow{6}{*}{$\begin{array}{c}\text { Mounted } \\
\text { mower } \\
\text { Posates + } \\
\text { Thresher } \\
\text { Mabrouk }\end{array}$} & \multirow{3}{*}{2.1} & 27.40 & & & & & & & & & & & & & & & & & & $*$ & & & & & \\
\hline & & & 31.41 & & & & & & & & & & & & & & & & & & & $*$ & & & & \\
\hline & & & 35.34 & & & & & & & & & & & & & & & & & & & & $*$ & & & \\
\hline & & \multirow{3}{*}{3.2} & 27.40 & & & & & & & & & & & & & & & & & & & & & $*$ & & \\
\hline & & & 31.41 & & & & & & & & & & & & & & & & & & & & & & $*$ & \\
\hline & & & 35.34 & & & & & & & & & & & & & & & & & & & & & & & $*$ \\
\hline
\end{tabular}




\section{RESULTS AND DISCUSSIONS}

\section{1- Crop variety and its characteristics}

Sample of 100 wheat plant SAKHA 93 variety were taken from random places of the field, plant height, stem diameter, grain moisture content, grain/straw ratio and the crop yield were measured, The averages values of this measurements were:

Plant height $98.9 \mathrm{~cm}$, stem diameter $3.5 \mathrm{~mm}$, grain moisture content $17.8 \%$, grain / straw ratio 1.09:1 and crop yield 2.73 ton grain / fed.

\section{2- Actual performance rate and field efficiency}

Table (3) shows that the highest significant actual performance rates resulted from the $1^{\text {st }}$ system (Multi-purpose combine harvester) at different forward and threshing speeds compared to other studied systems.

The highest actual performance rate $(0.73 \mathrm{fed} . / \mathrm{h}$ per meter cutting width) was achieved by the $1^{\text {st }}$ system (treatment E). This was followed by $3^{\text {rd }}$ system (treatment M), $2^{\text {nd }}$ system (treatment I) and $4^{\text {th }}$ system. The lowest actual performance rate $(0.26 \mathrm{fed} . / \mathrm{h}$ per meter cutting width) was attended by the $5^{\text {th }}$ system.

Increasing forward speeds of combine harvesters caused significant increment in actual performance rates. This was obvious as the increment of forward speed of $1^{\text {st }}$ system from 2.3 to $4.5 \mathrm{~km} / \mathrm{h}$ at 28.9 threshing drum speed caused an increase in actual performance by $155.3 \%$ (from 0.47 to $0.73 \mathrm{fed} / \mathrm{h}$ per meter cutting width).

It appears from Fig.(1) that the highest significant field efficiency $(94.3 \%)$ was obtained from the $4^{\text {th }}$ and $5^{\text {th }}$ systems, then $1^{\text {st }}$ system, $3^{\text {rd }}$ system and the lowest was from $2^{\text {nd }}$ system .

The field efficiency for the three combine systems decreased significantly by increasing the threshing drum speeds at the same forward speed. The $4^{\text {th }}$ and $5^{\underline{t}} \underline{\underline{h}}$ systems caused significant higher field efficiency compared to the combine systems since threshing takes place at a separate stage than that of the harvesting for the $4^{\text {th }}$ and $5^{\text {th }}$ systems. Also feeding rate of the threshers was at the recommended rate and the threshers were supplemented with 5 labors therefore there was little un-productive time. 
Table.(3): Actual performance rates under different wheat harvesting systems $(L S D=0.01)$

\begin{tabular}{|c|c|c|c|c|c|}
\hline $\begin{array}{c}\text { system } \\
\text { No. }\end{array}$ & $\begin{array}{c}\text { Forward } \\
\text { Speeds; } \\
\mathrm{Km} / \mathrm{h}\end{array}$ & $\begin{array}{c}\text { Threshing } \\
\text { drum } \\
\text { Speeds; } \\
\text { m /s }\end{array}$ & $\begin{array}{c}\text { Treatments } \\
\text { symbols }\end{array}$ & $\begin{array}{l}\text { Actual } \\
\text { performance } \\
\text { rate; fed/h }\end{array}$ & $\begin{array}{c}\text { Actual } \\
\text { performance } \\
\text { rate; fed/h } \\
\text { (per meter } \\
\text { cutting width) }\end{array}$ \\
\hline \multirow{6}{*}{1} & \multirow{2}{*}{2.3} & 28.90 & A & 1.98 & 0.47 \\
\hline & & 32.04 & B & 1.70 & 0.40 \\
\hline & \multirow{2}{*}{3.2} & 28.90 & $\mathrm{C}$ & 2.39 & 0.57 \\
\hline & & 32.04 & D & 2.30 & 0.55 \\
\hline & \multirow{2}{*}{4.5} & 28.90 & $\mathbf{E}$ & 3.06 & 0.73 \\
\hline & & 32.04 & $\mathbf{F}$ & 2.71 & 0.65 \\
\hline \multirow{4}{*}{2} & \multirow{2}{*}{1.8} & 22.70 & $\bar{G}$ & 0.59 & 0.29 \\
\hline & & 32.10 & H & 0.57 & 0.28 \\
\hline & \multirow{2}{*}{3.2} & 22.70 & I & 0.93 & 0.45 \\
\hline & & 32.10 & $\mathbf{J}$ & 0.88 & 0.43 \\
\hline \multirow{4}{*}{3} & \multirow{2}{*}{1.8} & 22.70 & $\bar{K}$ & 0.47 & 0.34 \\
\hline & & 32.10 & $\mathbf{L}$ & 0.39 & 0.28 \\
\hline & \multirow{2}{*}{3.2} & 22.70 & $\mathbf{M}$ & 0.74 & 0.53 \\
\hline & & 32.10 & $\mathbf{N}$ & 0.65 & 0.46 \\
\hline \multirow{3}{*}{4} & \multirow{3}{*}{1.8} & 27.48 & $\bar{O}$ & 0.31 & 0.31 \\
\hline & & 31.41 & $\mathbf{P}$ & 0.31 & 0.31 \\
\hline & & 35.34 & $\mathbf{Q}$ & 0.31 & 0.31 \\
\hline \multirow{6}{*}{5} & \multirow{3}{*}{2.1} & 27.40 & $\mathbf{R}$ & 0.31 & 0.26 \\
\hline & & 31.41 & $\mathbf{S}$ & 0.31 & 0.26 \\
\hline & & 35.34 & $T$ & 0.31 & 0.26 \\
\hline & \multirow{3}{*}{3.2} & 27.40 & $\mathbf{U}$ & 0.31 & 0.26 \\
\hline & & 31.41 & $\mathbf{W}$ & 0.31 & 0.26 \\
\hline & & 35.34 & $\mathbf{Y}$ & 0.31 & 0.26 \\
\hline
\end{tabular}

\section{3- Cutting efficiency}

It is obvious from fig. (2) that the highest significant cutting efficiency resulted from $2^{\text {nd }}$ system followed by the $4^{\text {th }}$ system, $3^{\text {rd }}$ system, $1^{\text {st }}$ system and $5^{\text {th }}$, respectively. The highest cutting efficiency averaged $(98.9 \%)$ as a result of $2^{\mathrm{ed}}$ system (treatments $\mathrm{G}$ and $\mathrm{H}$ ). However the significant lowest cutting efficiency resulted from $5^{\text {th }}$ ( treatments $\mathrm{U}, \mathrm{W}$ and $\mathrm{Y}$ ) as it averaged 
( $84.4 \%$ ) this may be due to the high vibration of harvesting toolbar at any change in soil level specially it is not controlled by a tractor.

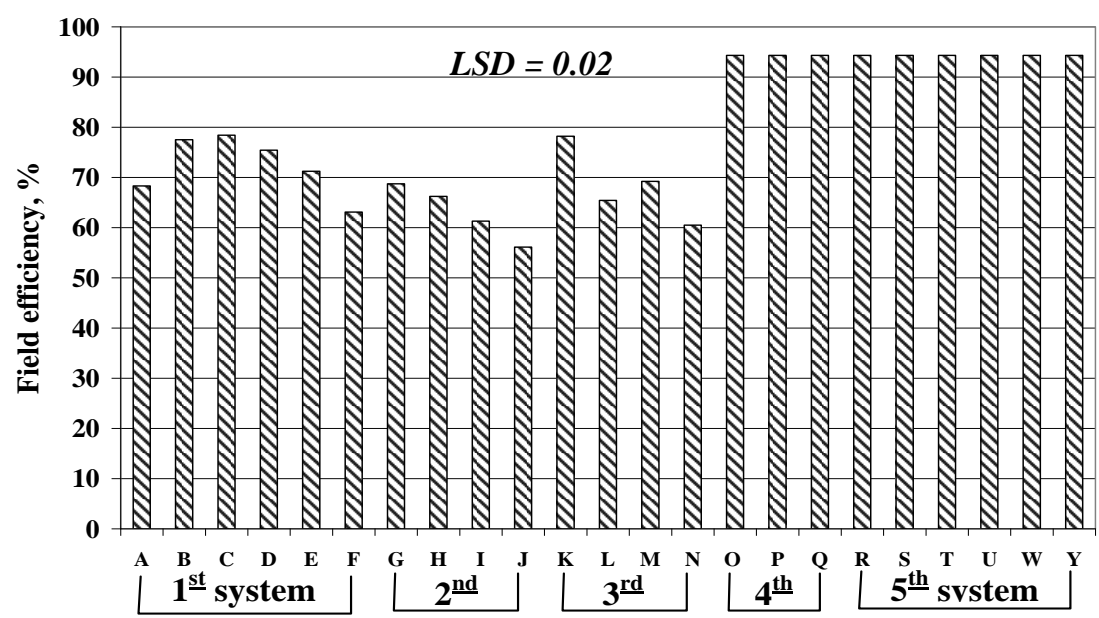

Treatments

Fig. (1): Field efficiency of the tested wheat harvesting systems.

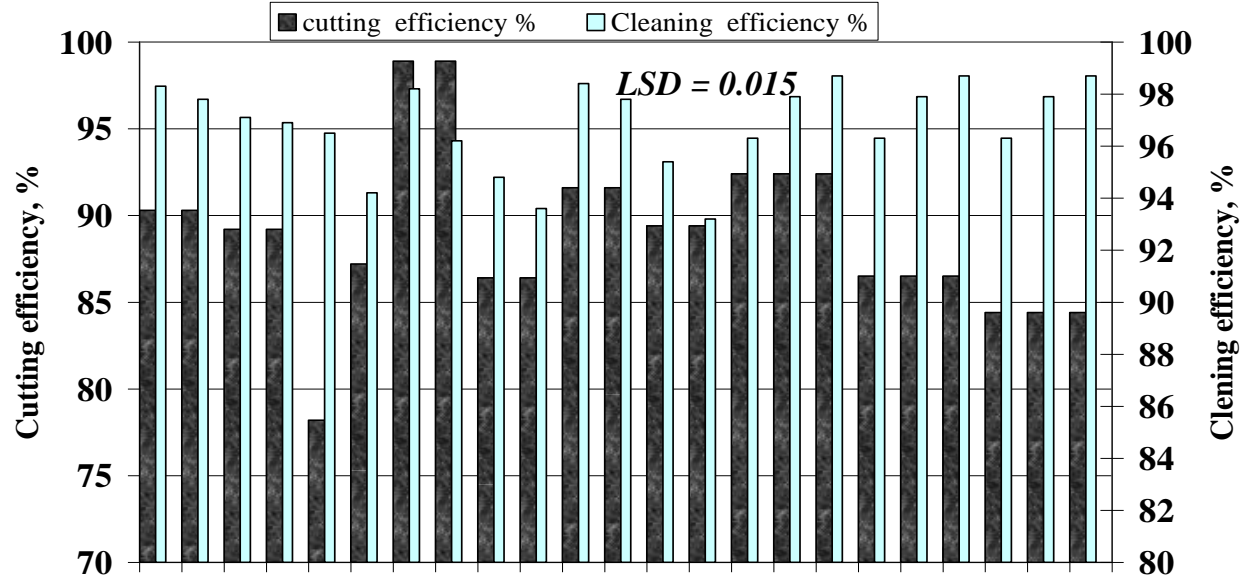

A B C D E F G H I J K L M N O P Q R S T U W Y

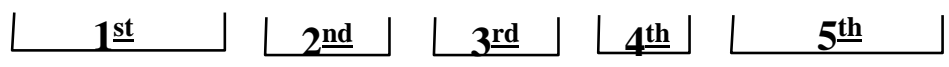

Treatments

Fig. (2): Cutting and cleaning efficiency of the tested wheat harvesting systems.

\section{4)- Cleaning efficiency}

Also, Fig (2) shows that the highest significant cleaning efficiency resulted from the three combine systems at lower forward and threshing drum speeds. On the other hand cleaning efficiency increased by increasing threshing drum 
speeds of the $4^{\text {th }}$ and $5^{\text {th }}$ systems. As threshing drum speed increases the speed of the separation fan increases which causes higher cleaning efficiency. The highest significant cleaning efficiency was $98.7 \%$ which resulted from $4^{\text {th }}$ and $5^{\text {th }}$ systems (treatments $\mathrm{Q}, \mathrm{T}$ and $\mathrm{Y}$ ).

\section{5) Percentage of total grain losses}

It is clear from fig (3) that the highest significant percentage of total grain losses averaged $8.82 \%$ resulted from the $5^{\text {th }}$ system (treatment $\mathrm{Y}$ ). This was followed by $4^{\text {th }}$ system, $1^{\text {st }}$ system, $2^{\text {nd }}$ system and $3^{\text {ed }}$ system, respectively. The highest percentage of total grain losses obtained by $5^{\text {th }}$ system might be referred to high compact of the plant stems by mower knives, and then they fall on the soil due to shattering effect.

The lowest significant percentage of total grain losses averaged 2.27\% resulted from $3^{\text {ed }}$ system ( treatment $\mathrm{K}$ ). Generally percentage of total grain losses increased by the increasing of forward speed. Since increasing forward speed causes increasing of the feeding rate, this leads to increasing the percentage of un-threshed grain. Also, increasing forward speed increases compact between wheat stems with harvester device which increases percentage of total grain losses. Increasing threshing drum speed leads to increasing number of grains compact during threshing process. Mean while increasing forward speed of combine harvesters $1^{\underline{\text { st }}}$ and $2^{\underline{\underline{n d}}}$ systems causes decreases in percentage of damaged grains. This might be due to increase in the plant stems feeding rate at higher forward speeds which decreases the compact effect consequently decreases percentage of damaged grains.

The lowest percentage of un-threshed grain $(0.51 \%)$ and damaged grains


significant percentage of grain losses averaged $1.12 \%$ which was obtained from the $4^{\text {th }}$ system ( treatments $\mathrm{O}, \mathrm{P}$ and $\mathrm{Q}$ ) and was not significantly different than that of $3^{\text {ed }}$ system ( treatments $\mathrm{K}$ ). The significantly lower percentage of total grain losses averaged $2.27 \%$ resulted from $3^{\text {ed }}$ system (treatments K ) 


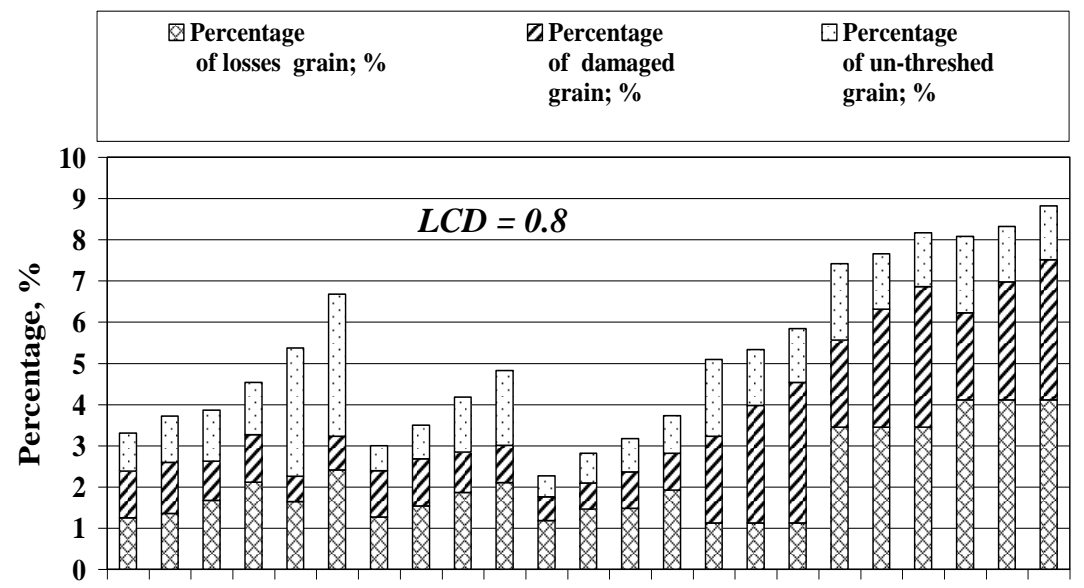

$\begin{array}{llllllllllllllllllllllll}\text { A } & \text { B } & \text { C } & \text { D } & \text { E } & \text { F } & \text { G } & \text { H } & \text { I } & \text { J } & \text { K } & \text { L } & \text { M } & \text { N } & O & \text { P } & \text { P } & \text { Q } & \text { R } & \text { S } & \text { T } & \text { U } & \text { W } & \text { Y }\end{array}$ L1st

\section{Treatments}

Fig. (3): Percentage of losses, damage and un- threshed grains of the tested wheat harvesting systems.

\section{6- Specific consumed energy}

Consumed energy was expressed as $\mathrm{kW} . \mathrm{h} / \mathrm{fed}$ and $\mathrm{kW} . \mathrm{h} / \mathrm{ton}$ and both had the same trend for the studied systems. From fig. (4) it is clear that the lowest significant consumed energy per fed. and per ton was achieved during operating the $1^{\text {st }}$ system followed by $3^{\text {rd }}$ system, $2^{\text {nd }}$ system, $4^{\text {th }}$ system and the $5^{\text {th }}$ system, respectively. This is due to high performance rate of $1^{\text {st }}$ system compared to other systems.

The specific consumed energy decreases by increasing forward speed with all systems. This is also due to higher performance rate at higher speeds. The higher consumed energy in the 4th and 5th systems than other systems is due to using two power sources for harvesting and threshing processes. The lowest significant consumed energy averaged $23.36 \mathrm{~kW} . \mathrm{h} / \mathrm{fed}$ which resulted from operation of the 1st system (treatment E). The general trend showed that for the same forward speeds the consumed energy increases by increasing threshing drum speed which may be referred to the increment in fuel consumption at higher threshing drum speed.

\section{7- Total cost and overall cost criterion}

Fig. (5) shows that forward and threshing drum speeds did not cause significant effect of the total cost and overall costs criterion for each of the 
five studied systems. However the lowest significant total cost (LE/h) resulted from $4^{\text {th }}$ system followed by both $3^{\text {rd }}$ and $5^{\text {th }}$ systems then the second system which was lower than the first system. On the other hand opposite

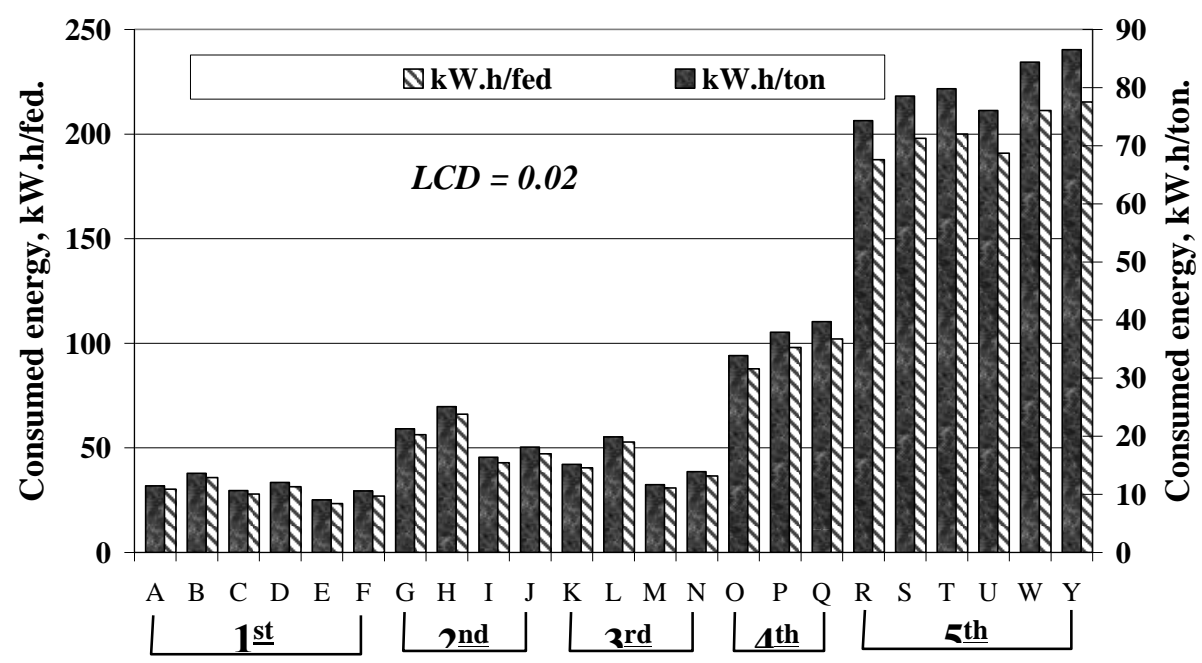

Treatment

Fig. (4): Specific consumed energy of the tested wheat harvesting systems.

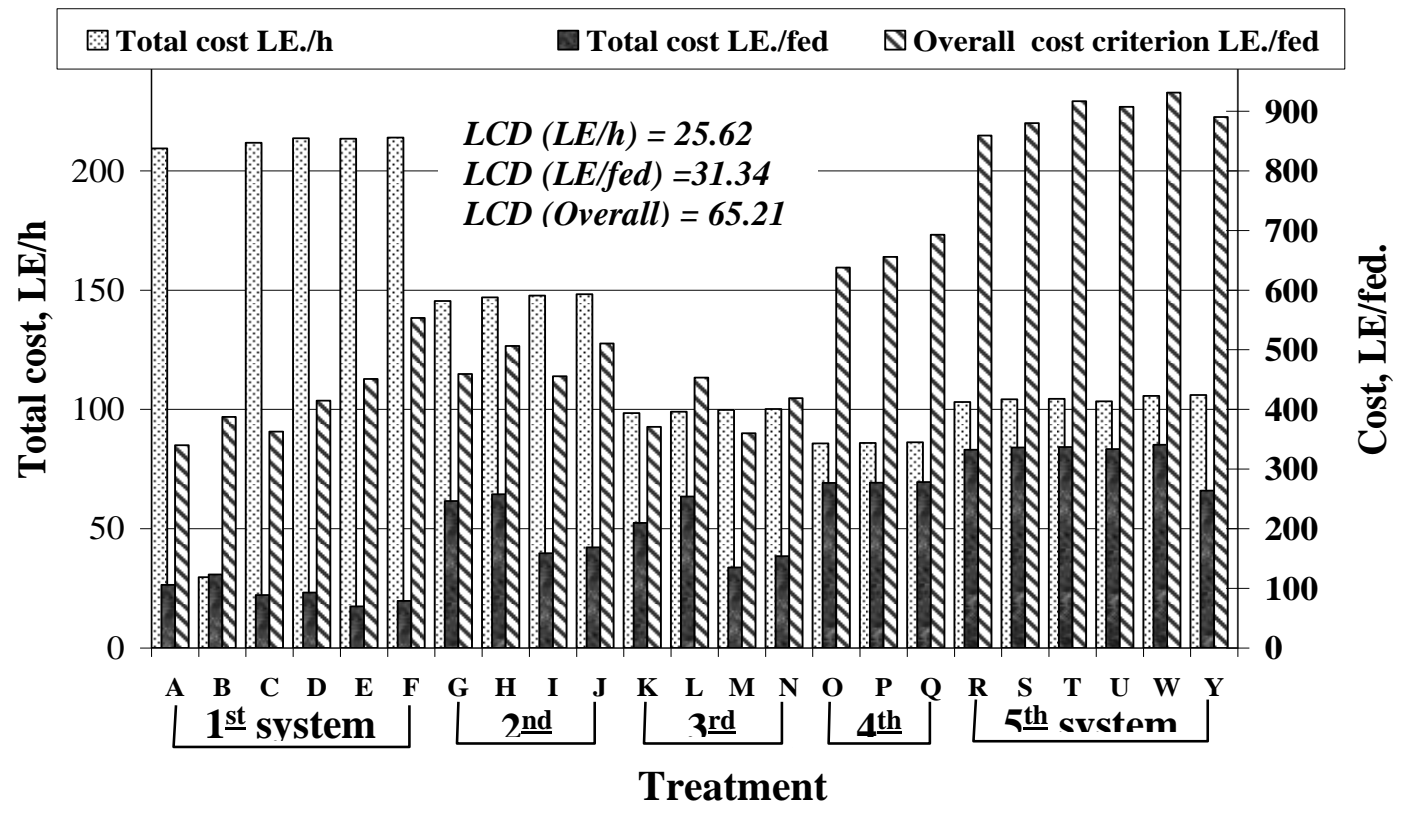

Fig. (5): Total cost and overall cost criterion of the tested wheat harvesting systems. 
trend of total cost (LE/fed) was observed for the studies system because the higher performance rates for the combine systems ( $1^{\underline{\text { st }}}, 2^{\text {nd }}$ and $\left.3^{\frac{\text { rd }}{}}\right)$ compared to $4^{\text {th }}$ and $5^{\text {th }}$ systems.

For the purpose of overall financial evaluation the optimum operating parameters (forward speed and threshing drum speed) were considered. The total cost of each system at its optimum working condition fall the following ascending order:

1 - $1^{\text {st }}$ system (Multi-purpose combine harvester) at $2.3 \mathrm{~km} / \mathrm{h}$ forward speed and $28.9 \mathrm{~m} / \mathrm{s}$ threshing drum speed.

2- $3^{\text {th }}$ system (Hold in combine harvester) at $3.2 \mathrm{~km} / \mathrm{h}$ forward speed and 22.7 $\mathrm{m} / \mathrm{s}$ threshing drum speed.

3- $2^{\text {nd }}$ system (Through in combine harvester) at $3.2 \mathrm{~km} / \mathrm{h}$ forward speed and $22.7 \mathrm{~m} / \mathrm{s}$ threshing drum speed.

4- $4^{\text {th }}$ system (Reaper + Thrasher) at $1.8 \mathrm{~km} / \mathrm{h}$ forward speed and $27.4 \mathrm{~m} / \mathrm{s}$ threshing drum speed.

5- $5^{\text {th }}$ system (mounted mower + Thresher) at $2.1 \mathrm{~km} / \mathrm{h}$ forward speed and $27.4 \mathrm{~m} / \mathrm{s}$ threshing drum speed.

\section{CONCLUSION}

From the results of the presents it can be recommended that:

1- Based on the studied performance and evaluation parameter ;

The highest performance rate was achieved by treatment $\mathrm{E}$ ( $1^{\text {st }}$ system at $4.5 \mathrm{~km} / \mathrm{h}$ forward speed and $28.9 \mathrm{~m} / \mathrm{s}$ threshing drum speed) ; The suitable cutting efficiency was realized by treatments $\mathrm{G}$ and $\mathrm{H}$ ( $2^{\text {nd }}$ system at 1.8 $\mathrm{km} / \mathrm{h}$ forward speed ) ; The highest cleaning efficiency was achieved by treatments $\mathrm{Q}, \mathrm{T}$ and $\mathrm{Y}\left(4^{\text {th }}\right.$ and $5^{\text {th }}$ systems at $35.34 \mathrm{~m} / \mathrm{s}$ threshing drum speed); and the lowest grain losses was attained by treatment $\mathrm{K}$ ( $3^{\text {ed }}$ system at $1.8 \mathrm{~km} / \mathrm{h}$ forward speed and $22.7 \mathrm{~m} / \mathrm{s}$ threshing drum speed).

2-According to the overall cost criterion it can be recommended to use Multi-purpose combine harvester in wheat harvesting.

\section{REFERENCES}

Afify, M. K., A. A. Nada and W. M. Mechail. (2000). Selection the proper system of planting and harvesting for rice crop. Misr J. Ag. Eng., 17 (2): $388-400$. 
El-Awady,M.N., G.H.El-Sayed A.H.Mohamed and I.Yehia. (2000). Evaluation of Barmel rice thresher. Misr J. of Ag. Eng., 17(3): 719-730.

El-Khateeb, H. A. (2005). A study on performance of axial flow combine harvester in rice crop harvesting. The $13^{\text {th }}$ Annual conference of the Misr society of Ag. Eng., 14-15 December 2005: 381-401.

El-Nakib, A.A., Z. Y. Abdel-Lateef, A. A. El-Messery and A. Khattab. (2003). Mechanical harvesting losses in rice crop using combine harvester. Misr J. Ag. Eng., 20 (4): 889-907.

El-Sharabasy, M. M. A. (2007). Total grain losses, energy and cost requirements for harvesting rice crops mechanically in delta Egypt. Misr J. Ag. Eng., 24 (1):1-17.

Arnaout, M. A., M. K. Abdel-Wahab and M. M. El-Sharabasy. (1998). Selecting the proper system for mechanizing grain crops harvesting in the small holdings. Misr J. Ag. Eng. 15 (1):133-144.

Ghonimy, M. I. and M. N. Rostom. (2002). Tech-economical approach to combine harvesters evaluation. Misr J. Ag. Eng., 19 (1): 83 - 99.

Kamel, O. M. (1999). Rice harvesting losses utilizing two different harvesting techniques of Japanese combine. Misr J. Ag. Eng.,16 (4): 237 $-251$.

Mohamoud M. A., M. M. El-Sharabasy and M. Kh. A. Khattab. (2006). Development of feeding device in a Turkish thresher machine. Misr J. Ag. Eng., 24(2): 235-258.

RNAM 1995. Test Code and Procedures for Agricultural Machinery. Technical Series 12, Regional Network for Agricultural Machinery (RNAM) of the United Nations. Philippines. PP: 227 -246.

Oida, A. 1997. Using personal computer for agricultural machinery management. Kyoto Univ. Japan. JICA Pub.

Omran, M. S. 1989. Comparative Study for Mechanical Methods of Waste Disposal in Dairy Farms-Free Open System. M Sc. Thesis , Fac. of Ag., Cairo Univ.

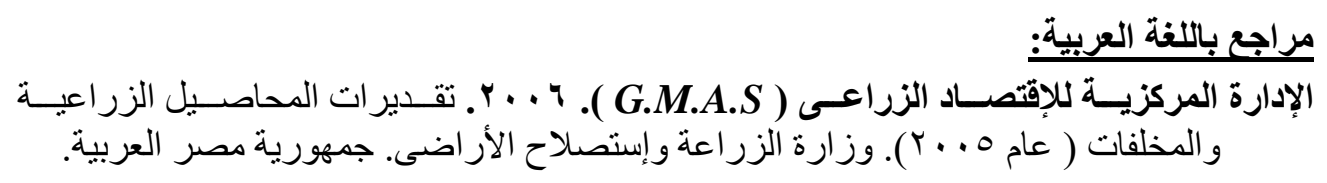


الملخص العربيى

\section{دراسة مقارنة لأكثر نظم حصاد القمح إنتشاراً فى مصر}

\section{محمد سيد عمران}

أجريت هذه الدر اسة فى مركز ميكنة الأرز - ميت الديبـة بمحافظـة كفر الثَيخ خـلال موسـم حصـاد

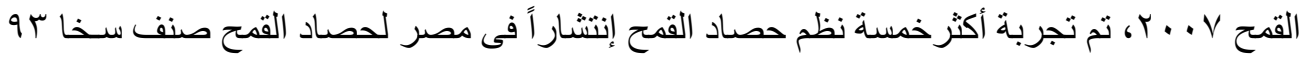

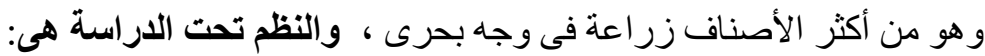

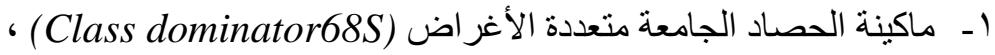

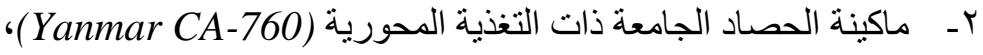

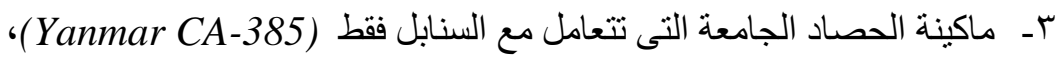

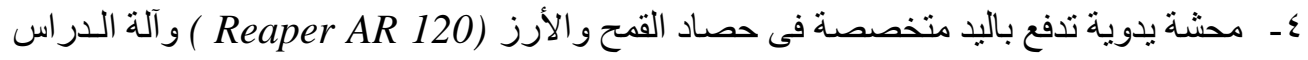

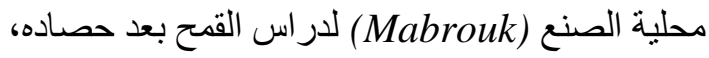
ــ محشة يدوية معلقة بالجرار ( Posates) و آلة الدر اس محلية الصنع (Mabrouk) لدر اس القمح بعد حصاده. وتم تقييم النظم وفقا للمعايير الفنية التالية (معدل الأداء ، الكفاءة الزمنية ، كفاءة القطع ، كفـاءة نظافـة الحبوب ، نسب الفو اقد فى الحبوب ، الطاقة المستهلكة) وكذللك المعيار المالى. وتم إستخدم إجمالى تكاليف حصاد الفدان كمعيار للمفاضلة بين النظم المختلفـة لحصـاد القــح تحـ

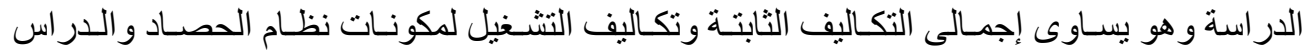

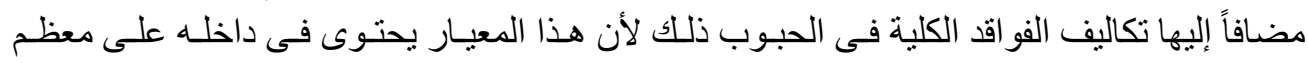

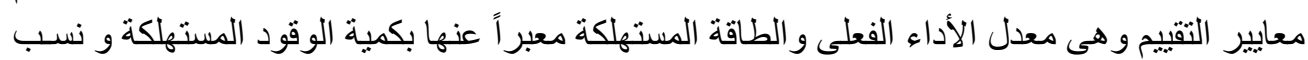

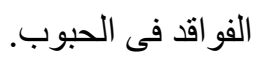

وأظهرت النتائج ما يلى ألى

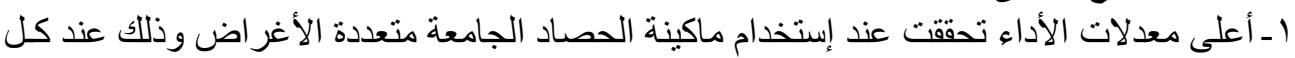

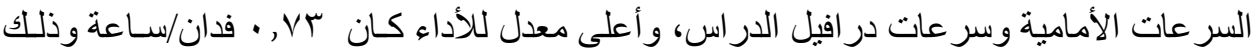

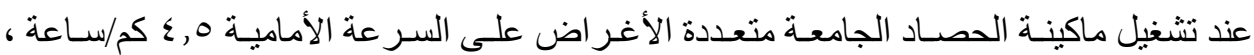

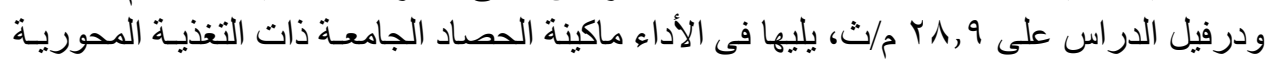

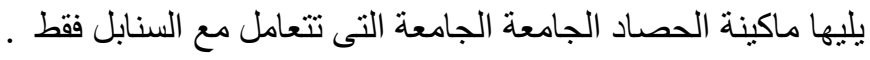
Y ـ أعلى كفاءة حقلية كانت عند تشغيل النظام الر ابع و الخامس ،يليهما ماكينة الحصاد الجامعة متعددة


تشغيل ماكينة الحصاد الجامعة ذات التغذية المحورية.

* مدرس بقسم الهندسة الزر اعية - زر اعة القاهرة. 


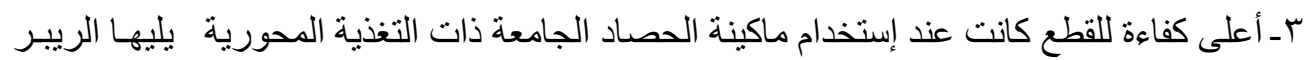

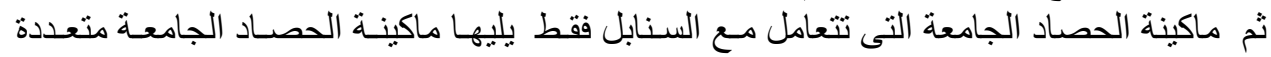

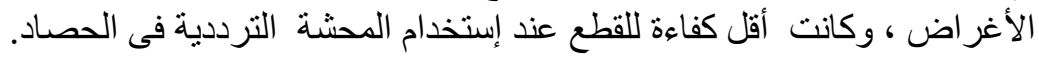

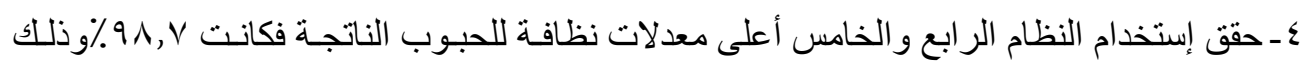

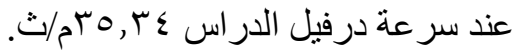

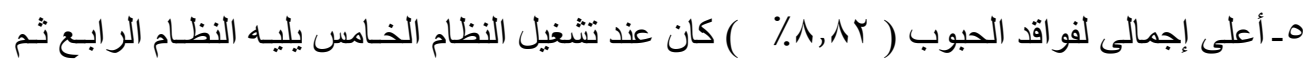

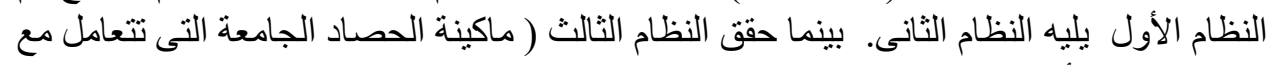

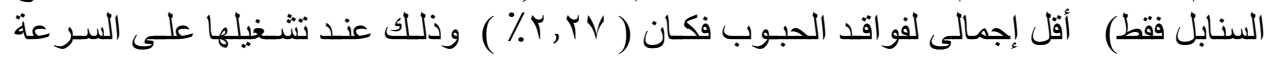

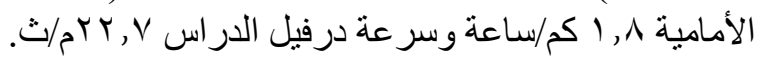

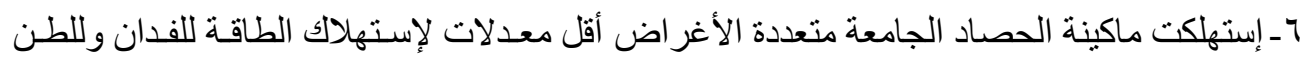

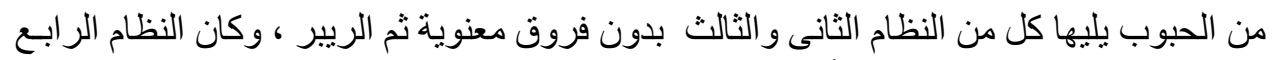
و الخامس من أعلى النظم إستهلاكاً للطاقية.

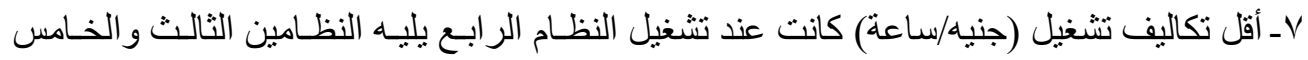

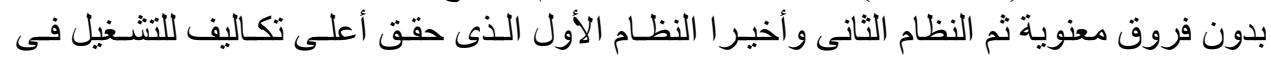

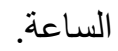

^- بإستخدام معيار المفاضلة (إجمالى تكاليف التشغيل مضافاً إليها تكلفة الفو اقد مـن الحبوب للفدان

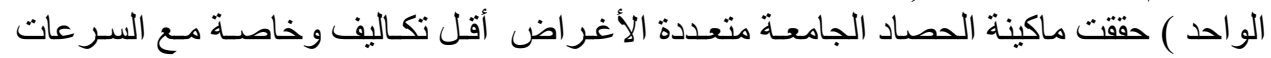

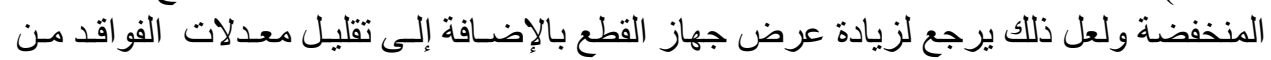
الحبوب.

\section{ومن هذه الاراسة يمكن الخروج بالتوصيات التالية: \\ ا - بالرجوع إلى معايير التقيم الفنية:}

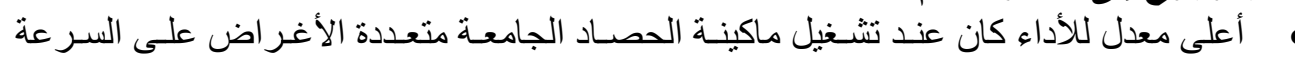

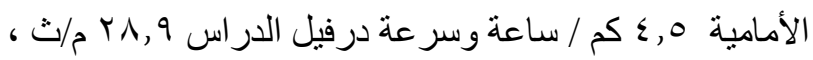

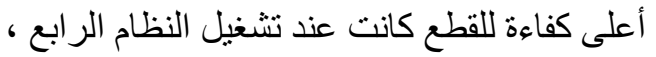

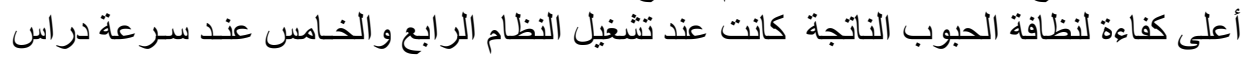

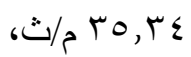

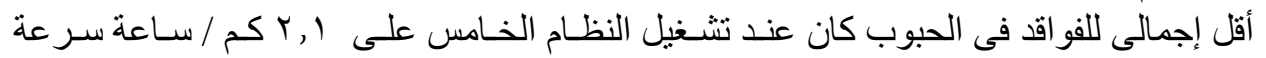

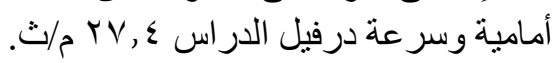
r-وبالرجوع لمعامل التقييم المالى العام: يكون إستخدام ماكينة الحصاد الجامعة متعددة الأغر اض اضل هى الأنسب لحصاد القمح. 